\title{
Qualitative assessment of the purity of multi-walled carbon nanotube samples using krypton adsorption
}

\author{
C.J. Gommes ${ }^{a}$, F. Noville ${ }^{a}$, C. Bossuot ${ }^{b}$, J.-P. Pirard ${ }^{a}$ \\ ${ }^{a}$ Laboratoire de Génie Chimique, Université de Liège B6a, B-4000 Liège, Belgium \\ ${ }^{\mathrm{b}}$ Nanocyl S.A., rue de l'Essor 4, B-5060 Sambreville, Belgium
}

\section{Abstract}

Krypton is a subcritical vapour at the nitrogen boiling temperature. As such, its adsorption on crystalline surfaces leads to condensation steps, typical of type VI isotherms according to IUPAC, while its adsorption on rough surfaces is BET-like. Based on this property of krypton adsorption at $77 \mathrm{~K}$, a methodology is proposed to determine the purity of carbon nanotubes samples. The method is tested on model samples obtained by mixing mechanically purified multi-walled carbon nanotubes with various amounts of the same catalyst as used for their synthesis.

\section{INTRODUCTION}

Since their discovery by Ijima in the early nineties as a byproduct of fullerene synthesis [1], carbon nanotubes have received a growing interest. A huge number of synthesis routes have been proposed, ranging from laser ablation of carbon target, catalytic chemical vapour deposition (CCVD), liquid phase synthesis, plasma methods, and so forth [2]. Also, a large variety of application niches have been identified that render nanotubes a promising material [3].

In order to meet the expected industrial needs for such materials, a large scale CCVD production facility has been developed in Nanocyl S.A., with a production capacity of the order of one $\mathrm{kg}$ of multi-walled carbon nanotubes (MWCNTs) per hour. In a typical CCVD reactor [e.g. 4], the contact between a gaseous hydrocarbon and an appropriate solid catalyst at high temperature results in the deposition of carbon nanotubes on the catalyst. The raw product that exits the reactor is therefore expected to contain carbon nanotubes, a catalyst residue and possibly amorphous carbon. The latter two products are usually referred to as impurities. A general method to assess the amount of impurities in a MWNT sample is not available yet. The present work reports on the use of krypton adsorption to determine the amount of nanotubes actually present in as-synthesized sample.

Krypton at $77 \mathrm{~K}$ is a subcritical vapour, the adsorption of which on crystalline surfaces is known to give rise to stepped isotherms, classified as type VI by IUPAC [5]. For such vapours, when a given pressure is reached the intermolecular forces between adsorbed molecules overwhelms their thermal energy, by which a phase transition occurs between and adsorbed gas-like and adsorbed dense phases $[6,7]$. This phenomenon leads to a riser in the isotherms that corresponds to the complete coverage, at a given pressure, of the surface by a 2D dense phase. Such isotherms have been reported for the adsorption of several subcritical vapours carbon nanotubes $[8,9]$. Since the monolayer condensation phenomenon is not expected for the adsorption on amorphous surfaces [7], we propose to exploit this 

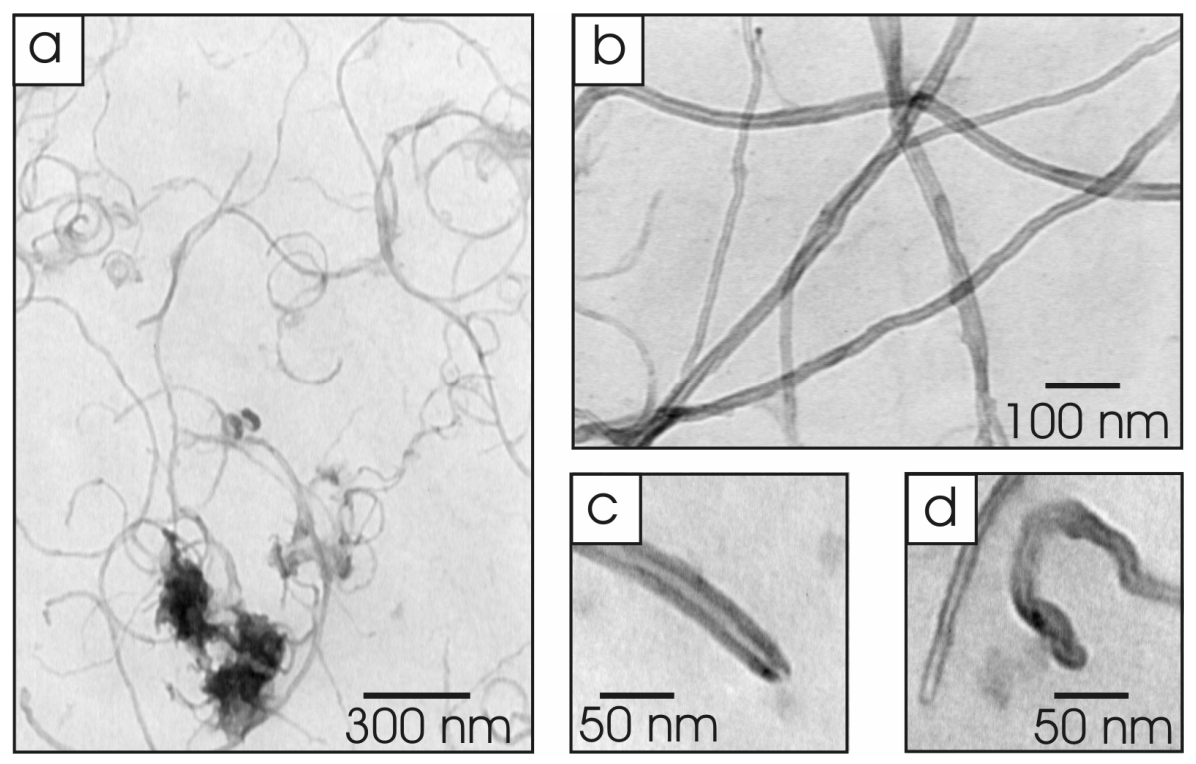

Figure 1. Example of transmission electron micrographs of the purified multi-walled nanotubes sample at various magnifications.

phenomenon to discriminate between the crystalline surface of the MWNTs and the amorphous surface of the impurities. The proposed methodology is tested on model samples obtained by mixing purified MWNTs with various amounts of the same catalyst as used for their synthesis.

\section{EXPERIMENTAL SECTION}

\subsection{Preparation and characterization of the samples}

The MWNTs are synthesized in a fixed bed CCVD reactor at $700^{\circ} \mathrm{C}$. A quartz boat containing about $1 \mathrm{~g}$ of catalyst is placed in the centre of the reactor fed with a $1-21(\mathrm{STP}) / \mathrm{min}$ flow of a 50-50\% mixture of nitrogen and ethylene. A typical reaction time is $20 \mathrm{~min}$. The used catalyst is $\mathrm{Fe}_{\mathrm{x}}-\mathrm{Co}_{\mathrm{y}}$ supported on alumina, prepared by impregnation as described elsewhere [10]. The purification of the MWNTs proceeds in two steps: (i) the sample is leached with concentrated fluoric acid in order to dissolve the catalyst support and the metallic particles; (ii) an acidic $\mathrm{KMnO}_{4}$ solution is used to selectively oxidize the amorphous carbon. After these treatments, the sample is filtered and washed with distilled water, and dried for $48 \mathrm{~h}$ in a vacuum oven heated at $120^{\circ} \mathrm{C}$.

Figure 1 displays several typical transmission electron micrograph of the purified sample, taken at various magnifications. As is visible in Figure 1a, a small amount of unidentified impurities are still present in the sample after the oxidation. At larger magnification, the hollow cavities of the tubes are visible as a bright line lying in their middle (Figure 1b-d). The question of whether the inner cavity of tube is accessible for adsorption cannot be cogently answered from TEM observations, as some tubes seem to be open (Figure 1c) while others seem to be closed (Figure 1d).

A series of 15 micrographs was acquired at the same magnification as Figure $1 \mathrm{~b}$, in order to assess the inner and outer diameter of the tubes. The micrographs were analyzed automatically using a digital image analysis procedure described elsewhere [11]. The obtained statistical distributions are plotted in Figure 2. The average values of the outer and inner diameters, together with their standard deviations are $d_{\mathrm{O}}=20 \pm 8 \mathrm{~nm}$ and $d_{\mathrm{I}}=6 \pm 3 \mathrm{~nm}$. 

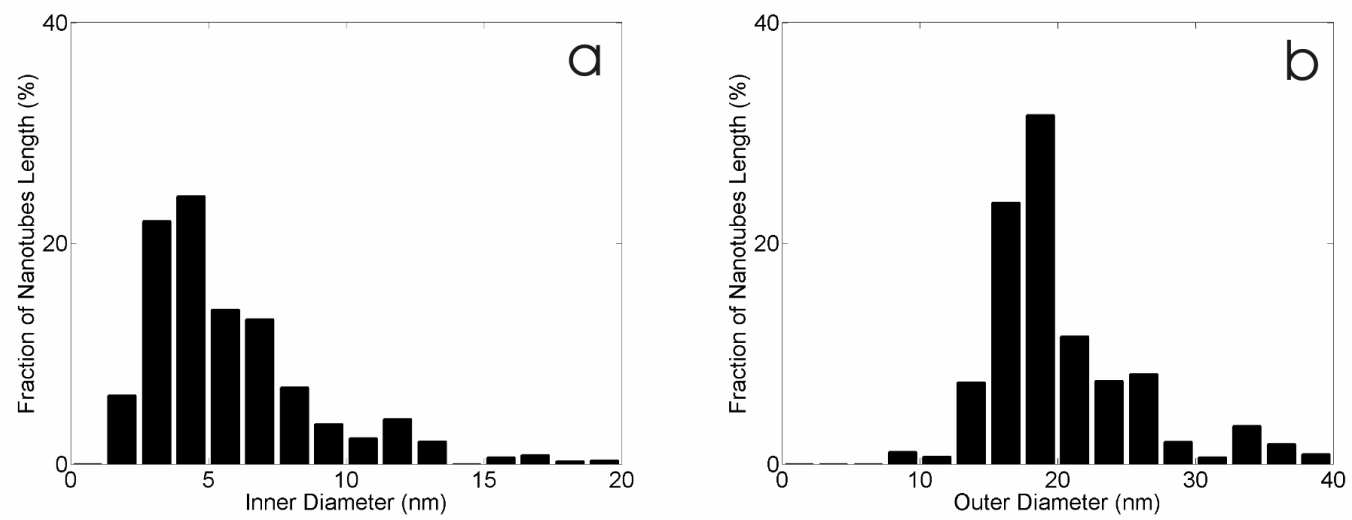

Figure 2. Statistical distributions of (a) the inner and (b) outer diameter of the nanotubes, estimated from digital image analysis of TEM micrographs.

Three mixtures were prepared by mixing mechanically the purified MWNTs materials with various amounts of catalyst. The nomenclature of the samples is given as $X(Y)$, where $Y$ is the weight percentage of nanotubes.

\subsection{Krypton and nitrogen adsorption measurements}

The krypton and nitrogen adsorption isotherms are determined at nitrogen boiling temperature by the classical volumetric method with a CE Instruments SORPTOMATIC 1990 series of THERMO ELECTRON. The device is equipped with an additional 10 torr pressure gauge and a turbomolecular pump. The used high purity krypton $(99.997 \%)$ and nitrogen (99.999 \%) were purchased from Air Liquide.

The samples were first outgassed at $10^{-4} \mathrm{~Pa}$ during $16 \mathrm{~h}$, then heated from $25^{\circ}$ to $150^{\circ} \mathrm{C}$ at a rate of $1^{\circ} \mathrm{C} / \mathrm{min}$ and kept at $150^{\circ} \mathrm{C}$ during $12 \mathrm{~h}$. The weight loss of each sample during this treatment was determined and removed from the mass of the samples. Typically, the isotherms were determined on $150 \mathrm{mg}$ of sample. Preliminary experiments allowed establishing the optimal experimental conditions to obtain reproducible isotherms starting at a relative pressure of $10^{-4}$.
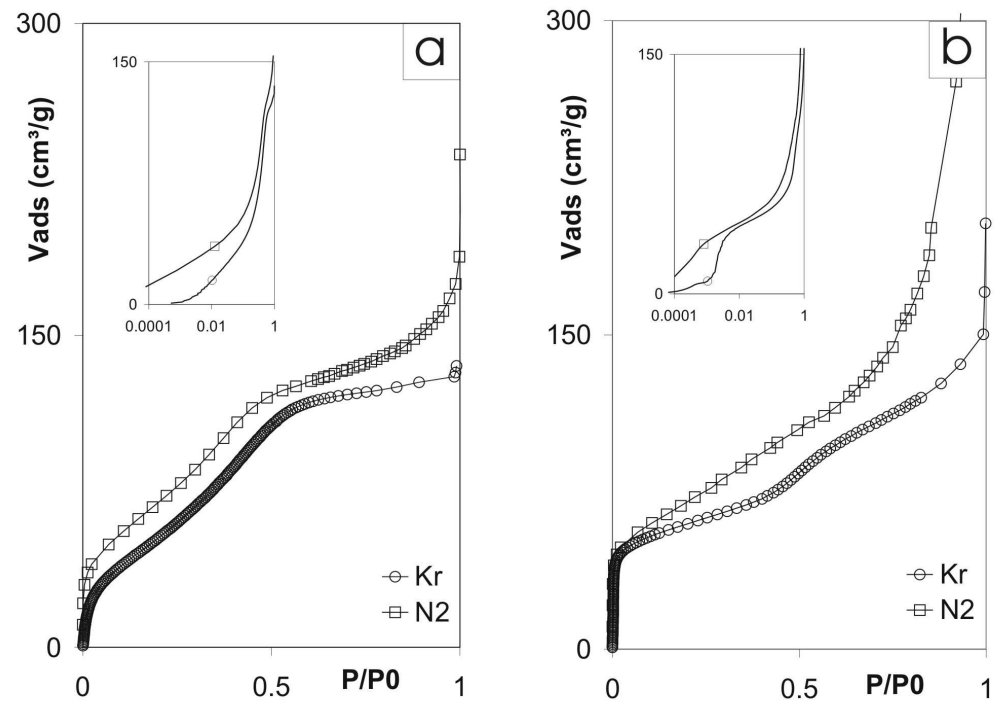

Figure 3. Krypton and nitrogen adsorption isotherms on the catalyst (a) and on the nanotubes (b). The insets magnify the low pressure adsorption with a logarithmic scale. 


\section{RESULTS AND DISCUSSION}

\subsection{Qualitative description of the isotherms}

Adsorption of $\mathrm{Kr}$ and $\mathrm{N}_{2}$ on the catalyst leads to isotherms with a very similar shape (Figure 3a). A type IV isotherm is obtained in both cases, with a strong adsorption near $P / P_{0}$ 0.4 , characteristic of very small mesopores [5]. On the contrary, qualitatively different isotherms are obtained for $\mathrm{Kr}$ and $\mathrm{N}_{2}$ adsorbed on the nanotubes (Figure $3 \mathrm{~b}$ ). The shape of the $\mathrm{N}_{2}$ adsorption isotherm is reminiscent of a type II isotherm, typical of a nonporous or macroporous solid, while the adsorption of $\mathrm{Kr}$ leads to type VI stepped isotherms. The origin of the steps in type VI isotherms is the occurrence of a phase transition in the adsorbed layer, between two adsorbed gas-like and dense phases [7], which phenomenon can be qualitatively captured by a 2D van der Waals equation for the adsorbate [6]. The first step, that is visible in the inset of Figure $3 \mathrm{~b}$, corresponds to the condensation of the first $\mathrm{Kr}$ monolayer on the surface of the nanotubes, and the second step near $P / P_{0}=0.5$ corresponds to the condensation of a second monolayer on the top of the first one [7,8].

Figure 4 displays the $\mathrm{Kr}$ and $\mathrm{N}_{2}$ isotherms on the whole series of samples. Figure $4 \mathrm{a}$ clearly highlights the fact that the krypton condensation step is a characteristic of nanotubes. For nitrogen adsorption, only a slight hump is visible, whose amplitude increases when increasing the nanotubes content of the samples. A similar isotherm has been reported for long for the adsorption of $\mathrm{N}_{2}$ on well graphitized surfaces and its shape is sometimes referred to as the Joyner-Emmet step [12]. It corresponds to the completion of the first adsorbed layer, which is progressive because adsorbed nitrogen is supercritical at $77 \mathrm{~K}$ [7].

The finite width of the adsorption steps of $\mathrm{Kr}$ contrasts with what is observed on planar graphite, in which a neat adsorption vertical riser is present [e.g. 7]. Also, as observed by other authors [8], the pressure at which the krypton monolayer condensation occurs is higher on carbon nanotubes than on graphite. This shift originates in the positive curvature of the adsorbent surface, which hinders adsorption. Since the condensation of krypton on thick nanotubes should occur at a lower pressure than on thin nanotubes, the polydispersity of the samples can broaden the adsorption step. The width of the adsorption step of $\mathrm{Kr}$ on nanotubes could therefore be related the width of the diameter distribution of the tubes.
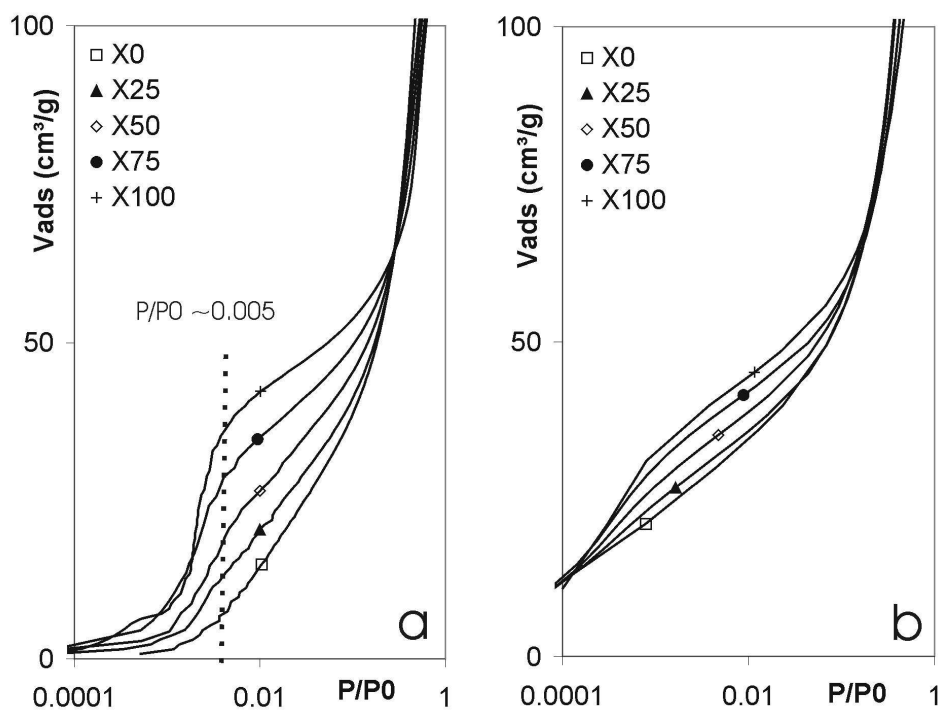

Figure 4. Krypton (a) and nitrogen (b) adsorption isotherms on the samples with increasing nanotube purity (X0 to X100). 


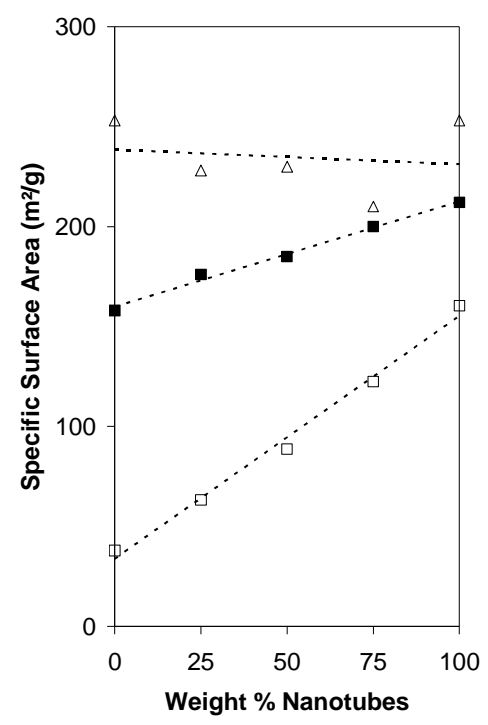

Figure 5. Specific surface areas estimated from the BET model applied to the krypton and nitrogen adsorption, $S_{\mathrm{Kr}}(\square)$ and $S_{\mathrm{N} 2}(\Delta)$, and from the height of the krypton condensation step, $S_{\text {step }}(\square)$.

\subsection{Quantitative analysis of the isotherms}

Although this model is not suited for analyzing stepped adsorption, the $\mathrm{Kr}$ and $\mathrm{N}_{2}$ isotherms were fitted with the standard BET model [6]. The estimated specific surface areas with the two different vapours are referred to hereafter as $S_{\mathrm{Kr}}$ and $S_{\mathrm{N} 2}$. They were estimated using the standard values for the surface covered by a single molecule $16.2 \AA^{2}$ for $N_{2}$ [5] and $15.7 \AA^{2}$ for $\mathrm{Kr}[7,8]$. These surfaces are plotted in Figure 5 as a function of the nanotubes content of the samples. There is an important scattering of the estimated surface areas (especially for $\mathrm{N}_{2}$ ) that probably stems from the fact that the BET model could only be applied to the adsorption data over a very narrow pressure range. The very linearity of the plots in Figure 5 suggests that the specific surface area of any sample be the average of the specific surface areas of the catalyst and nanotubes, weighed by their relative mass fractions. However, as is visible in this figure, the specific surface area of the catalyst is very close to that of the nanotubes, so that the BET surface area cannot serve as a measure of the nanotubes content of the samples.

As already mentioned stepped isotherms are generally observed for the adsorption of subcritical vapours on crystalline surface [7]. Since the nanotubes are the only species with a crystalline surface in the studied samples, the height of the step should be associated with the presence of nanotubes, which is confirmed by Figure 4a. In order to make this statement more quantitative, the height of the krypton adsorption step is estimated as the amount adsorbed at $P / P_{0}=510^{-3}$. The choice of the pressure limit of the step is arbitrary as it only results from the visual inspection of the isotherms. This approximation however proofs to be useful for an order of magnitude analysis of the adsorption process. The so estimated volume of the step is converted to the specific area $S_{\text {step }}$ of the surface on which stepped adsorption occurs, as described elsewhere [13]. This latter quantity is plotted in Figure 5.

$S_{\text {step }}$ is a linear function of the of the weight fraction, $y$, of purified nanotubes in the sample, with parameters

$S_{\text {step }}\left(\mathrm{m}^{2} / \mathrm{g}\right)=34+122 y$ 
The line does not pass through the origin because the adsorption on the pure catalyst is such that the amount adsorbed on it at $P / P_{0}=510^{-3}$ account for ca. $34 \mathrm{~m}^{2} / \mathrm{g}$. Moreover, the height of the step for the purified sample corresponds to $156 \mathrm{~m}^{2} / \mathrm{g}$, while the total specific surface of the same sample is $S_{\mathrm{Kr}}=212 \mathrm{~m}^{2} / \mathrm{g}$. Since the BET surface $S_{\mathrm{Kr}}$ accounts for the total specific surface area, and $S_{\text {step }}$ accounts only for the crystalline fraction of the same surface, the difference between these two numbers could mean that there is still a significant amount of impurities left in the sample. This would be confirmed by the TEM micrograph of Figure 1a. Another origin of this difference could be that the acidic treatment used to purify the sample significantly damaged the surface of the nanotubes. A previous study showed that this would result in a smoothing of the adsorption step [14].

Another possibility to explain the difference between $S_{\mathrm{Kr}}$ and $S_{\text {step }}$ of the purified sample is that the nanotubes are so entangled in sample that only a fraction of their surface is accessible for layer by layer adsorption. To gain some insight into this issue, it is interesting to compare $S_{\text {step }}$ to the geometrical surface of the tube. The nanotubes can be modelled as ideal hollow cylinders with inner and outer diameters $d_{\mathrm{I}}$ and $d_{\mathrm{O}}$, and made of a material with density $\rho$. This results in the following surface over mass ratios [13]

$$
\frac{S_{T}}{m}=4 \frac{d_{O}+d_{I}}{\rho\left(d_{O}{ }^{2}+d_{I}{ }^{2}\right)} \quad \frac{S_{O}}{m}=4 \frac{d_{O}}{\rho\left(d_{O}{ }^{2}+d_{I}{ }^{2}\right)}
$$

where $S_{\mathrm{T}}$ and $S_{\mathrm{O}}$ stand for total and outer surface. Using the diameters estimated from image analysis (section 2.1) and assuming that the nanotubes wall has the same density as graphite with $\rho \sim 2 \mathrm{~g} / \mathrm{cm}^{3}$, leads to $S_{\mathrm{T}} / m=143 \mathrm{~m}^{2} / \mathrm{g}$ and $S_{\mathrm{O}} / m=110 \mathrm{~m}^{2} / \mathrm{g}$. Although these figures have to be considered as orders of magnitude, it can be noticed that the outer surface of the nanotubes alone could hardly account for the value of $S_{\text {step }}$. However, the total surface compares well with the height of the step. It seems therefore that the inner surface of the tubes be accessible for adsorption. Furthermore, it is likely that the difference between $S_{\mathrm{Kr}}$ and $S_{\text {step }}$ stems from the presence of impurities, rather than from the entanglement of the tubes.

\section{CONCLUSION}

Krypton adsorption can be used to qualitatively assess the purity of multi-walled carbon nanotubes samples. The exploited phenomenon is the krypton monolayer condensation that occurs exclusively on the crystalline surface of the nanotubes but not on the catalyst residue, nor on amorphous carbon. In principle, the same methodology could be used with the adsorption of any vapour below its 2D critical temperature. On the contrary, this kind of analysis is not feasible with $2 \mathrm{D}$ supercritical vapours, such as $\mathrm{N}_{2}$ at $77 \mathrm{~K}$, for which the step is replaced by a mere hump.

The proposed methodology was tested on a series of model samples obtained by mixing mechanically a purified nanotubes sample with known amounts of catalyst. It was shown that the height of the first condensation step in the $\mathrm{Kr}$ adsorption isotherm is indeed directly related to the amount of nanotubes in the sample. Comparing the height of the adsorption step with the geometric surface of the nanotubes further suggests that the purified nanotubes sample still contained a significant amount of impurities, probably amorphous carbon. 
Acknowledgements C.J. Gommes is grateful to the National Funds for Scientific Research (FNRS, Belgium) for a Ph.D. research fellow position. The authors thank the Ministère de la Région Wallonne (DGTRE) and the Ministère de la Communauté Française de Belgique (Action de recherche concertée 00/05-265) for their financial support.

\section{REFERENCES}

[1] S. Ijima, Nature 354 (1991) 56.

[2] C. Journet, P. Bernier, Appl. Phys. A 67 (1998) 1.

[3] Nanoscience and Nanotechnologies: Opportunities and Uncertainties. London: The Royal Society \& The Royal Academy of Engineering, 2004.

[4] C. Gommes, S. Blacher, C. Bossuot, P. Marchot, J. B.Nagy, J.P. Pirard, Carbon 42 (2004) 1473.

[5] J. Rouquerol D. Avnir, C.W. Fairbridge, D.H. Everett, J.H. Haynes, N. Pernicone, J.D.F. Ramsay, K.S.W. Sing, K.K. Unger, Pure Appl. Chem. 66 (1994) 1739.

[6] D.D. Do, Adsorption Analysis: Equilibria and Kinetics, Series in Chemical Engineering, Volume 2, Imperial College Press, London, 1998.

[7] A. Thomy, X. Duval, J. Regnier, Surf. Sci. Rep. 1 (1981) 1.

[8] K. Masenelli-Varlot, E. Mc Rae and N. Dupont-Pavlovsky, Appl. Surf. Sci. 196 (2002) 209.

[9] A. Bougrine, N. Dupont-Pavlovsky, J. Ghanbaja, D. Billaud and F. Beguin, Surf. Sci. 506 (2002) 137.

[10] K. Hernandi, A. Fonseca, J. B.Nagy, D. Bernaerts, A. Fudala, A.A. Lucas, Zeolites 17 (1996) 416.

[11] C. Gommes, S. Blacher, K. Masenelli-Varlot, C. Bossuot, E. McRae, A. Fonseca, J. B.Nagy, J.-P. Pirard, Carbon 41 (2003) 2561.

[12] L. Joyner, P. Emmet, J. Am Chem Soc., 70 (1948) 2353.

[13] C. Gommes, S. Blacher, N. Dupont-Pavlovksy, C. Bossuot, D. Marguillier, A. Fonseca, J. B.Nagy, J.P. Pirard, Colloid Surf. Sci. A 241 (2004) 155.

[14] R. Babaa, E. McRae, C. Gommes, S. Delpeux, G. Medjahdi, S. Blacher, F. Beguin, American Institute of Physics Conference Proceedings 723 (2004), 133. 